\title{
Circuit
}

Musiques contemporaines

\section{Le festival présences 99 : une fenêtre ouverte sur la nouvelle réalité musicale québécoise}

\section{Jean Boivin}

Volume 10, numéro 2, 1999

Les racines de l’identité

URI : https://id.erudit.org/iderudit/004654ar

DOI : https://doi.org/10.7202/004654ar

Aller au sommaire du numéro

Éditeur(s)

Les Presses de l'Université de Montréal

ISSN

1183-1693 (imprimé)

1488-9692 (numérique)

Découvrir la revue

Citer ce compte rendu

Boivin, J. (1999). Compte rendu de [Le festival présences 99 : une fenêtre ouverte sur la nouvelle réalité musicale québécoise]. Circuit, 10(2), 39-50. https://doi.org/10.7202/004654ar d'utilisation que vous pouvez consulter en ligne. 


\title{
CHRONIQUE «ÉCLATS ET RÉSONANCES »
}

\section{Le festival Présences 99 : une fenêtre ouverte sur la nouvelle réalité musicale québécoise}

\author{
Jean Boivin
}

L'Amérique du Nord, dont nombre de représentants viennent étudier à Paris et s'y confronter à leurs homologues européens, regarde depuis longtemps vers l'Europe et la France. Depuis peu la réciproque est vraie [...]

(Yves Prin, programme de Présences 99, p. X.)

Du 29 janvier au 14 février 1999, la création musicale québécoise let plus spécifiquement montréalaise) a été à l'honneur à Paris. En effet, le festival, qui en était à sa neuvième édition, s'articulait cette année autour de trois pôles, qui sont autant de centres de création musicale : Paris - New York - Montréal. Le volet parisien comprenait une importante rétrospective du (relativement) jeune compositeur français Pascal Dusapin ', dont plusieurs œuvres pour diverses formations, incluant une création, furent entendues. Quatre formations différentes ont également rendu hommage à Gérard Grisey, décédé subitement en 1998. Les accents particuliers placés sur New York et Montréal ont permis à des ensembles nord-américains de présenter une sélection d'œuvres de compositeurs rarement joués en Europe. Pour certains, surtout les plus jeunes, il s'agissait d'une première exécution à Paris. Les parcours biographiques des compositeurs 
de notre époque étant souvent fort sinueux, les nationalités les plus diverses se côtoyaient en fait dans les programmes. En tout, 62 compositeurs, 21 concerts, dont 3 avec grand orchestre, tous gratuits et radiodiffusés, en direct ou en différé, par Radio France et de nombreuses radios dans le monde, y compris la chaîne culturelle de Radio-Canada ${ }^{2}$. Le Québec était représenté par 21 compositeurs. Leurs œuvres étaient réparties entre 9 concerts, dont 5 leur étaient entièrement consacrés. La Société de musique contemporaine du Québec et le Nouvel Ensemble Moderne ont présenté chacun 2 concerts, regroupés dans les tout premiers jours du festival (entre le 29 janvier et le 4 février inclusivement) ${ }^{3}$. J'ai eu la chance de pouvoir y assister, ainsi qu'à une table ronde qui regroupait huit compositeurs québécois et deux représentants de la Société Radio-Canada. Ce texte se veut un écho de cet événement unique dans I'histoire de la musique au Québec, et une réflexion plus large sur l'impact de la musique québécoise récente en France.
2. Sur les ondes de Radio-Canada, quatre émissions successives de la Grande soirée du mercredi, ont été consacrées au festival Présences 99, soit les mercredis 3, 10, 17 et 24 février 1999.

3. Une œuvre électroacoustique de Francis Dhomont, Frankenstein Symphony (1997), construite à l'aide de fragments d'œuvres de 22 amis québécois du compositeur, dont plusieurs ex-étudiants, occupait entièrement le cinquième concert, produit par INAGRM. D'autres œuvres de compositeurs québécois figuraient au programme de concerts présentés par le quatuor Arditti (Provost), l'Orchestre philharmonique de Radio France (Cherney et Longtin, dans deux concerts distincts) et le Chœur de Radio France (Vivier et Mather).

\section{Une programmation bien assumée}

D'abord, quelques mots sur la portion de la programmation qui touche plus particulièrement le Québec. Les 21 compositeurs et les 24 œuvres ont été choisis, en concertation, par Denys Bouliane, coordonnateur artistique de la participation québécoise ${ }^{4}$, Yves Prin, directeur artistique de Présences, Lorraine Vaillancourt et Walter Boudreau, respectivement directeurs artistiques du Nouvel Ensemble Moderne et de la Société de musique contemporaine du Québec. Au premier coup d'œil, on constate que, si plusieurs catégories d'âge étaient représentées, une prédominance était accordée aux compositeurs nés entre 1947 et 1957 Isoit, en ordre alphabétique, Linda Bouchard, Walter Boudreau, Denys Bouliane, Michel Gonneville, Denis Gougeon, Jean Lesage, Isabelle Panneton, Serge Provost, Claude Vivier). Venait immédiatement ensuite, en importance numérique, le groupe des Brian Cherney, José Evangelista, Michel Longtin et John Rea, leurs aînés de quelques chandelles à peine. Seuls trois compositeurs nés avant 1940 ont été retenus: Bruce Mather, Gilles Tremblay et Francis Dhomont. Dans l'autre plateau du balancier, quatre compositeurs nettement plus jeunes: Sean Ferguson, Estelle Lemire, Ana Sokolovic et Michael Oesterle ${ }^{5}$. Bien sûr, cette "froide " répartition ne signifie pas grand-chose en soi, si ce n'est l'absence, voulve et assumée, de tous les compositeurs, à l'exception de Gilles Tremblay, ayant joué un rôle de premier plan dans la naissance de ce qu'il est convenu maintenant d'appeler l'avant-garde musicale québécoise. D'une part, les Garant, Mercure, Pépin, Morel, Hétu, Prévost, Saint-Marcoux et autres ne s'inscrivent pas dans le groupe d'âge qu'on avait choisi de mettre de l'avant. D'autre part, l'influence de l'Europe aura été assurément pour ceux-là déterminante: "les voies ouvertes par Varèse,
4. L'origine de cette participation du Québec au festival Présences remonte aux premiers contacts entre le compositeur Simon Bertrand et Yves Prin. Denys Bouliane a pris la relève à l'automnme 1996, a été désigné officiellement coordonnateur de la participation québécoise en avril 1997 et a mené à son terme l'ambitieux projet.

5. Les noms de Denys Bouliane et de Claude Vivier apparaissaient à deux reprises dans les programmes, deux de leurs œuvres ayant été données, dans des concerts différents. Leur aîné Bruce Mather était également représenté par deux œuvres, plutôt anciennes en fait: La lune mince, pour chœur, de 1965, et Ausone, de 1979, la seconde donnée en création française. 
Messiaen, Boulez, Dutilleux, Honegger, Stravinsky ou la Seconde École viennoise ont été parcourues en tous sens", écrivait à ce propos Denys Bouliane dans le programme détaillé préparé par Radio France (1999, p. XXIII) ${ }^{6}$. Si ce dernier précise qu'il serait «bien mesquin » de reprocher aux pionniers, qui ont dû mettre les bouchées doubles pour rattraper un important retard, une certaine orthodoxie $^{7}$, cette page de notre histoire récente semble bel et bien tournée. Du moins la voulaiton telle.

Un festival, à plus forte raison s'il est international, n'a pas bien sûr à se substituer à une leçon d'histoire. La sélection des compositeurs et des œuvres a été délicate et difficile, l'espace disponible se révélant somme toute assez restreint. Le comité de programmation a donc voulu mettre l'accent sur ce qui distingue depuis une ou deux décennies la musique québécoise de celle de l'Ancien monde ${ }^{8}$. Chose certaine, on a cherché à préciser cette identité québécoise (voilà un thème à la mode au Québec !) et, surtout, à en montrer les différentes facettes. Dans le texte déjà cité, Bouliane insiste sur l'important renouvellement stylistique apporté par les compositeurs retenus et "dont les moyens d'expression sont en accord avec leurs aspirations et avec les paradoxes de la société québécoise» (ibid.). Maintenant en accord, fautil comprendre, ou enfin en accord.

Cette nouvelle société québécoise est donc émancipée et plurielle, davantage même que ne le laisse entendre Bouliane dans son texte. Les notices biographiques des compositeurs sélectionnés montrent en effet une diversité de parcours et d'allégeance esthétique tout à fait caractéristique de la réalité québécoise, ou plutôt montréalaise, des 25 dernières années: plusieurs de ces compositeurs ne sont pas nés au Québec, n'y ont pas reçu l'essentiel de leur formation musicale, mais ont choisi d'y vivre et d'y poursuivre leur œuvre de création tout en s'engageant intensément dans la vie musicale elle-même. Fort heureusement, l'art n'a que peu à faire de ce que le politique désignera comme le Québécois «pure laine». Rappelons que l'ensemble de la programmation du festival Présences montrait bien ce décloisonnement frappant des différentes cultures. Quant à la présence des jeunes étoiles montantes, québécoises ou autres, elle est tout à I'honneur des organisateurs de ce festival qui ont rendu hommage au cours des éditions antérieures aux "valeurs sûres " de la scène internationale que sont Ligeti, Berio, Kagel et Xenakis, tout en offrant une tribune largement médiatisée aux nouvelles tendances.

L'espace nous manque pour nous attarder sur le détail de la programmation. Trois créations mondiales (de Panneton, Bouliane et Provost, dans ce dernier cas une commande de Radio France) côtoyaient des œuvres en majorité assez récentes, données pour la plupart en création française. On remarquait toutefois quelques partitions plus anciennes, comme les deux œuvres de Vivier, le compositeur québécois le plus connu sur la scène internationale ${ }^{9}$, Demain les étoiles de Walter Boudreau (1981), les deux pièces de Mather déjà mentionnées ${ }^{10}$ et La route des pélerins reclus de Michel Longtin (1984). Une première ébauche du
6. Le texte de Bouliane, intitulé «À l'est de l'ouest, à l'ouest de l'est, cherchez au nord! » a été repris, en plus développé, dans un document préparé pour l'occasion, Présence de la musique québécoise. Vingtdeux portraits instantanés (1999, p. 5-11). Le sous-titre "Toile de fond d'une création musicale en pleine effervescence » s'ajoutait alors au titre déjà donné.

7. Ibid., p. xxiii du programme de Radio France et p. 9 du document québécois. 8. Par exemple, la musique de Garant serait, de ce point de vue, trop près de celle de Boulez et de la Seconde École viennoise pour apporter quelque chose de vraiment neuf au public français. Les programmations régulières de la $S M C Q$ et du NEM ne font par ailleurs que peu de place, en temps normal, aux œuvres représentatives composées au Québec durant les années cinquante et soixante, et pour ainsi dire jamais à celles qui relèvent d'une esthétique néo-classique ou néotonale. II n'est donc aucunement surprenant que la participation de ces organismes au festival Présences 99 reflète ces choix esthétiques déjà bien établis. On peut cependant déplorer qu'il n'y ait guère d'autres options en ce sens au Québec. En effet, plusieurs œuvres marquantes de notre histoire musicale récente ne soit jamais entendues en concert et les enregistrements sur disque dont on dispose sont souvent anciens et peu satisfaisants sur le plan technique et artistique.

9. En fait foi ce détail significatif. Une table, sur laquelle était présentée de la documentation concernant la musique québécoise, avait été disposée à l'entrée de l'Auditorium Olivier-Messiaen de Radio France où avaient lieu les concerts. Les exemplaires du numéro double que Circuit consacrait en 1991 à Claude Vivier (vol. 2 $n^{\circ}$ 1-2) ont été les premiers à s'envoler, de même que le coffret Claude Vivier de disques compacts de l'Anthologie de la musique canadienne (ACM 36 CD 1-4, 1990). Les deux œuvres de Vivier à avoir été données au cours du festival sont Ô! Kosmos pour chœur (1973) et Trois airs pour un opéra imaginaire (1982).

10. Voir la note 5. 
projet montre que l'on aurait souhaité pouvoir entendre à Paris plusieurs œuvres pour orchestre (Berliner Momente III de Boudreau, Orion de Vivier, Passaglia ohne Thema de Bouliane), mais elles furent finalement remplacées, sans doute pour des considérations budgétaires et en raison de la disponibilité réduite des larges ensembles français, par d'autres œuvres destinées à des effectifs plus réduits ${ }^{11}$. Par contre, et malgré des mesures d'économie tout à fait légitimes, l'ampleur de l'événement a tout de même permis quelques «folies » qui en soulignaient justement le caractère exceptionnel. C'est ainsi que le Gamelan Evergreen Club de Toronto, un ensemble de huit musiciens, est venu à Paris expressément pour jouer une seule pièce, L'arbre de Borubudur de Gilles Tremblay, pour gamelan et ensemble instrumental, tandis que Demain les étoiles de Walter Boudreau exigeait la présence sur scène de douze saxophonistes. Le spectaculaire et efficace Train pour l'enfer de Denis Gougeon, écrit pour les Percussions de Strasbourg, a été interprété par ce même ensemble, qui se joignait pour l'occasion à celui de la SMCQ. Dans les trois cas, l'exécution fut à la mesure des attentes. (À notre humble avis toutefois, Boudreau a composé de meilleures œuvres depuis, mais peutêtre pas de plus «rebelles" et de plus typiquement nord-américaines, Boudreau se réclamant ici de sa formation de saxophoniste de jazz et de son expérience au sein du groupe rock l'Infonie.)
1 1. La concertation s'étendit au partage des ressources, puisqu'une demi-douzaine d'interprètes ont joué avec les deux ensembles, qui se sont en fait croisés à Paris, l'ensemble de la SMCQ étant arrivé à Paris le jour même du départ du NEM.

\section{Une fête pour les Québécois, mais un impact limité sur le public français}

Pour les compositeurs qui ont pu assister à l'événement (heureusement un bon nombre d'entre eux, grâce à une aide spéciale du Conseil des arts et des lettres du Québec), ce fut, comme le résumait si bien Isabelle Panneton sur les ondes de la radio de Radio-Canada, une "véritable fête " (La grande soirée du mercredi, chaîne culturelle FM de Radio-Canada, 10 février 1999). Tant de concerts de qualité en succession, mettant en vedette des interprètes de très haut calibre, présentés devant un public attentif et généralement nombreux, voilà qui a de quoi redonner de l'énergie à un créateur. Lorraine Vaillancourt y voit la preuve que la "mort du public» tant annoncée n'est pas pour demain (ibid.).

Hélas, et c'est là qu'il faut revenir sur terre, le public visé, placé devant une forte concentration d'événements 11 concerts en 9 jours, entre le 29 janvier et le 6 février), s'est présenté en moins grand nombre aux 4 concerts consacrés à la musique québécoise qu'aux grandes soirées en hommage à Dusapin ou à Grisey, où l'auditorium Olivier-Messiaen était plein à craquer ${ }^{12}$. Et les critiques parisiens, partagés chaque soir entre une foule d'événements prestigieux ly compris, cette semaine-là, une première à l'Opéra de Paris) ne donnèrent que peu
12. Plusieurs centaines de personnes se sont quand même présentées. Les concerts du NEM, en apparence fort bien placés le premier week-end du festival, étaient en fait coincés entre la soirée d'ouverture, très glamour, largement consacrée à Dusapin, et cet hommage à Grisey, qui réunissait également le Tout-Paris musical intéressé par ce répertoire (ou venu pour "y être vu»...) 
d'échos à cette visite préparée avec tant de soin. Peut-être est-il aussi pertinent de noter que peu de compositeurs français de renom ont assisté aux concerts où les œuvres québécoises étaient présentées, ce qui en dit long sur la réalité de cette "ligne d'échanges singulièrement fertiles tracée entre Ancien et Nouveau Monde", annoncée par Pascal Dumay et Jean-Michel Nectoux dans le programme de Radio France (op. cit., p. VII) ${ }^{13}$. Quant aux Américains, ils n'étaient tout simplement pas encore arrivés à Paris à cette date...

J'aurais voulu, tout au long de ces quelques jours, pouvoir entendre ces musiciens familiers et ces œuvres, parfois aussi assez familières ${ }^{14}$, avec des oreilles vierges, et une autre culture que la mienne. Pouvoir entendre les commentaires du public, après l'audition d'une œuvre aussi exigeante et virtuose que Demain les étoiles de Walter Boudreau (certains ont dû, pour paraphraser cet auditeur de la création de la Troisième symphonie de Beethoven, vouloir crier par moment: "Vingt francs pour que cela s'arrête!»|. Denys Bouliane, interrogé par Mario Paquet en vue de la rediffusion des deux concerts présentés par le NEM, n'était aucunement dupe de la situation. II lui semblait tout à fait normal que certains auditeurs européens aient eu du mal à comprendre et à apprécier la musique qui leur était présentée. Une musique qui s'éloigne consciemment, selon lui, de "certains canons de la modernité française ou allemande " de cet "espéranto » musical qui a pu être rêvé par un Boulez, un Stockhausen au début des années cinquante, au-delà de toutes considérations nationales. "[ll est] très important de prendre conscience que notre affirmation culturelle passe par la foi en certaines idées [...], en certaines de nos actions. [Important de] croire que notre différence peut s'articuler dans le sonore, et la proposer ${ }^{15}$.»

De fait, le critique du Monde, Pierre Gervasoni, connu pour sa plume acerbe, n'a pas été séduit outre mesure et titrait son unique article, au lendemain du premier concert du NEM, Postmodernisme québécois à Radio France (3 février 1999, p. 29). II note que le Nouvel Ensemble Moderne aurait pu, pour l'occasion, "changer son nom en Nouvel Ensemble Postmoderne, tant les œuvres proposées [...] affichaient un retraitement licencieux [sic] de références historiques »! Tout en relevant au passage les qualités particulières, et notamment l'esprit ludique, des œuvres de Jean Lesage (Les sensations confuses), de Michel Gonneville (Chute-Parachute) et de Denys Bouliane (Du fouet et du plaisir), il concluait que les Québécois "privilégie[nt] le contenu au détriment de la forme pour un résultat anecdotique ${ }^{16}$ ". Denys Bouliane réagira à cette critique, au micro de Radio-Canada que lui tendait Mario Paquet.

En fait il faut comprendre que le mot postmodernité, dans les milieux de la musique contemporaine français ou allemand, est péjoratif. [...] En Amérique du Nord, on parle de postmodernité comme d'un phénomène de société, ce que c'est, en fait. Les châteaux forts de la modernité de la musique contemporaine sont rébarbatifs à cette idée, parce qu'[elle remet] en question justement les millions qui ont été consacrés à édifier ces châteaux forts de la modernité. Qu'est-ce que [...] la modernité,
13. Il est important de noter que la rencontre initialement prévue entre un groupe de compositeurs québécois et Gérard Grisey n'a pas eu lieu, puisque ce dernier est décédé subitement en novembre 1998. L'événement devait avoir lieu au Conservatoire national de musique de Paris, dans la classe de composition dont Grisey était le titulaire et en présence de ses élèves. II est regrettable qu'aucune autre tribune officielle d'échanges, outre le traditionnel cocktail offert lors de la soirée d'ouverture, n'ait été organisée pour remplacer ce qui aurait sans aucun doute été un moment mémorable pour tous les intervenants.

14. Je pense par exemple à ChuteParachute de Michel Gonneville, interprétée par Jacques Drouin, le pianiste du Nouvel Ensemble Moderne.

15. C'est moi qui souligne. (La grande soirée du mercredi, 10 février 19991.

16. Curieusement, John Rea a prophétiquement déclaré, dans une entrevue fictive publiée dans le document Présence de la musique québécoise: "En Allemagne, notre musique manque de contenu, me semble-t-il ; et jouée en France, hélas, elle manque de style» ( p. 78). 
rapidement? C'est [...] croire que la musique a un pouvoir auto-intrinsèque, c'est-àdire qu'elle se suffit à elle-même. La postmodernité est la remise en question de ces idées, et une [...] tentative de se reconnecter avec du symbolique, du culturel, du "malpropre», de l'imprécis, du subjectif. Et ça, quand, pendant 45 ans, on a édifié un temple, des temples, et des structures qui soutiennent cette idée de pureté lqui se réfère [...] à l'après-guerre, c'est extrêmement important de [le] comprendre, parce que le pouvoir politique y a été mêlél [...]

On imagine que monsieur Gervasoni se sent dépositaire de la modernité, son article semble le laisser croire. Alors il voit d'un mauvais ceil que des gens, pas encore établis au niveau international, disons des "sans-culottes 》 peut-être à son point de vue! [...] se mêlent de proposer autre chose, et de basculer un petit peu ce socle que nous, on trouve un peu trop rigide. On n'est pas les seuls!

(La grande soirée du mercredi, 17 février 1999)

\section{Une table ronde à la fois exaspérante et instructive}

Venons-en à un moment qui me semble particulièrement significatif de l'idée que l'on peut se faire, en France notamment, de la vie musicale au Québec. II s'agit de la table ronde radiophonique, animée par Jean-Michel Damian dans le cadre de son émission hebdomadaire Les imaginaires, et regroupant un bon nombre des compositeurs québécois qui ont pu se rendre à Paris. Denys Bouliane, Brian Cherney, Denis Gougeon, Sean Ferguson, Michel Gonneville, Isabelle Panneton Serge Provost et John Rea y participaient, ainsi que deux représentants de la Société Radio-Canada: Laurent Major, réalisateur et coordonnateur de la musique contemporaine aux émissions culturelles, et Mario Paquet, animateur de l'émission La Grande soirée du mercredi, dans le cadre de laquelle les concerts de Présences 99 dont il est question ici ont été diffusés. Le thème de cette rencontre était, bien entendu, la création musicale québécoise. L'émission de deux heures et demie comprenait de larges extraits musicaux ${ }^{17}$ et la discussion se déroulait en direct, devant un public peu nombreux ll'émission avait lieu par un beau samedi après-midi) mais attentif.

D'entrée de jeu, il était manifeste que l'animateur (et réalisateur) Jean-Michel Damian ne s'était aucunement préparé, en dépit de l'abondante documentation qui lui avait été fournie. De plus, ses connaissances de la géographie canadienne et de sa diversité socioculturelle se sont révélées fort limitées. Son étonnement non dissimulé devant le fait que trois anglophones (Rea, Cherney et Ferguson) se trouvaient là, s'exprimant dans un français correct et échangeant sur
17. On peut se demander à quand remonte une semblable mise à l'honneur des propos de nos compositeurs dans les médias électroniques québécois... Certes la chaîne culturelle de Radio-Canada était partie prenante de Présences 99 et a même diffusé à la radio certains concerts en direct ou en différé rapproché, mais les portions d'entrevues avec les créateurs ont depuis plusieurs années été réduites au minimum sur les ondes de la radio d'État, sans doute par crainte d'effaroucher la célèbre let imaginaire) "Dame de la rue Panet», dont le bien-être est si cher à la haute direction de cette institution et à laquelle Gilles Archambault dédiait il y a quelques mois une chronique vitriolique et justifié (Le Devoir, 20 avril 1999, p. A7). À titre de comparaison et encore une fois au crédit de Radio France, je soulignerai qu'au cours de cette première semaine du festival Présences 99, Pascal Dusapin était l'invité d'une émission quotidienne d'une durée de deux heures et demie sur les ondes de France Musique, où il parlait de sa musique et de celle qui le stimule. 
un ton on ne peut plus courtois avec leurs collègues francophones, rappelait les gaffes cocasses, mais ô combien exaspérantes, de tant d'intervieweurs d'émissions françaises de variété, fort mal documentés sur la réalité québécoise et canadienne. On pourrait aussi s'attarder sur les nombreux problèmes techniques et autres maladresses de réalisation survenus au cours de l'émission. Plus regrettables paraissent, hélas, les relents d'attitude impérialiste, voire colonialiste, souvent perceptibles dans les questions ou commentaires de Jean-Michel Damian, par exemple lorsqu'il était question de la formation complémentaire que nombre de compositeurs québécois, depuis Rodolphe Mathieu et Claude Champagne, sont allés chercher en France, ou de la rassurante "hexagonalité » de leurs thèmes d'inspiration (l'intérêt de Denys Bouliane pour la poésie de Boris Vian, la passion de Bruce Mather pour les grands crus français, etc.). Certes, il est tout à fait normal qu'un animateur cherche à relier le thème de l'émission en cours avec le vécu culturel de ses auditeurs - le Québec n'est-il pas en ce sens presque aussi exotique pour les Français que la Pologne ou la Chine, dixit Damian? - mais le fait est que plusieurs participants, face à la superficialité des questions posées et du ton plutôt condescendant adopté par l'animateur, ont eu du mal à dissimuler leur malaise sur la scène, tout comme les quelques Québécois qui se trouvaient dans la salle. D'ailleurs, aucun des participants à qui j'en ai reparlé n'a gardé un bon souvenir de cette émission où n'auraient été échangées que des banalités.

Pourtant, au-delà de ces "irritants», ce qui nous intéresse en premier lieu dans cette table ronde, c'est le portrait du Québec actuel qui s'en dégageait. Car $c^{\prime}$ était bien là le propos premier de l'émission ${ }^{18}$. Les liens privilégiés entretenus par plusieurs compositeurs québécois actifs, à la veille de la Révolution tranquille, avec de grands compositeurs et pédagogues français, par exemple avec Olivier Messiaen, ne pouvaient être passés sous silence. Ainsi, l'importante influence de Gilles Tremblay ressortit clairement des propos d'Isabelle Panneton et de Serge Provost, mais ce dernier fit toutefois valoir que la personnalité des compositeurs et pédagogues québécois de cette génération était telle que lui-même sentit le besoin de s'exiler un temps en Europe et cela, afin de se trouver, de définir ses priorités de compositeur. Afin, aussi, d'être en contact avec d'autres modèles que ceux transmis par Tremblay à ses élèves. Notons qu'il en allait de même pour Garant, dont on connaît bien les prises de position en faveur de la modernité européenne ${ }^{19}$.

À cet égard, Bouliane dira que Vivier, l'éternel absent de ces débats, fit dans les années soixante-dix l'effet d'un véritable pavé dans la mare puisqu'il exprima clairement sa distance face aux modèles et ne participa pas au discours, alors extrêmement polarisé, entre les tenants du modernisme et ceux qui favorisaient un style plus accessible. II proposa plutôt des chemins nouveaux, en s'abreuvant par exemple à des sources orientales. Dans le même ordre d'idées, Laurent Major est d'avis que l'influence d'un Xenakis, mis à l'écart par le Domaine musical, a été plus grande au Québec qu'en France. II y a été reçu avec les mêmes égards que
18. L'animateur eut apparemment souhaité retracer toute l'histoire culturelle du Québec depuis la Conquête. II fallut le sens de l'àpropos de Laurent Major et de Denys Bouliane pour que la discussion commence aux années cinquante, sans s'y attarder lourdement. En effet, huit compositeurs se trouvaient là, dont la démarche, ainsi qu'un extrait substantiel d'une de leurs œuvres, devaient être présentés au public; 150 minutes n'y suffiraient pas, et les derniers à parler durent se contenter de bien peu de temps d'antenne..

19. Voir notamment l'article signé par l'auteur de ces lignes, "La classe de composition de Serge Garant, ou le sentier de la lucidité », dans Circuit, vol. 7, n² 1996, p. 37-55. 
Boulez, et y est revenu plusieurs fois, notamment à l'occasion de la création du Polytope de Montréal, en $1967^{20}$. En fait, une grande liberté dans le "traitement» des influences ressortait clairement du corpus d'œuvres présentées à Paris ${ }^{21}$

La présence de compositeurs d'âge, de culture et d'orientation stylistique différents a fait en sorte qu'un pan très large de la réalité musicale québécoise actuelle fut couvert, au moins rapidement, dans le cours de l'émission. Ainsi, le rôle joué par les François Morel, Jacques Hétu et André Prévost ne fut pas oublié, ni l'impact des interventions régulières de Serge Garant au micro de Radio-Canada (il y démontrait sans relâche, de dire Michel Gonneville, que "la musique du Québec n'est pas celle des sapins et du sirop d'érable...»|. Fut aussi noté le soutien essentiel apporté par les institutions, gouvernementales ou autres, tels le Conseil des Arts du Canada (calqué non pas sur un modèle français, mais sur le British Council, et donc indépendant du point de vue politique) ou encore la Société Radio-Canada lqui, de préciser Laurent Major, accorde 12 commandes par année à des compositeurs, sans toutefois qu'il soit possible de commander une œuvre à un compositeur étranger, alors que Serge Provost avait reçu une commande de Radio France destinée à être jouée par le quatuor Arditti).

La concentration d'interprètes exceptionnels, dévoués à la musique de notre siècle, fut bien sûr soulignée, de même que le désintérêt déplorable de la majorité des orchestres symphoniques, fort peu disposés à jouer la musique nouvelle en dehors d'un cadre bien circonscrit ${ }^{22}$. L'Orchestre symphonique de Montréal, l'un des premiers ambassadeurs de la vie musicale québécoise à l'étranger, a été alors pointé du doigt et accusé de ne pas remplir son mandat. Composer de la musique orchestrale pose donc aux jeunes compositeurs, tel Sean Ferguson, des difficultés réelles. D'où, selon un participant, l'urgence de s'exprimer, de dire, plutôt que de s'enfermer dans de stériles spéculations théoriques. Denys Bouliane insista à ce propos sur la «réapparition » de la communication dans la musique québécoise récente, et notamment dans celle de Denis Gougeon, qu'il qualifia de «raconteur ».

Quant aux trois anglophones déjà nommés, ils apportaient un point de vue complémentaire et fort intéressant - en plus d'être en lien direct avec le thème sous-jacent de ce numéro de Circuit - puisqu'ils ont tous trois choisi le Québec après avoir amassé ailleurs une large part de leur bagage musical. John Rea qualifiera même de "privilège » le fait d'avoir reçu une troisième formation en se transplantant dans le milieu culturel particulièrement riche qu'est le Québec. Un milieu dont la diversité et l'ouverture contrastent avec ceux de Toronto ou Winnipeg, comme Yves Prin l'aurait lui-même constaté. Brian Cherney, qu'on devinera plutôt discret pendant ces discussions, revendiquera lui aussi un héritage multiforme, qui va de Debussy (toujours aussi important pour lui à ce jour) à George Crumb, en passant par Lutoslawski et aux séjours qu'il fit à Darmstadt et à Munich à la fin des années soixante. Quant à Sean Ferguson, installé au
20. Voir à ce sujet le numéro de Circuit consacré à Xenakis (vol. 5, n², 1994), et où l'on trouve notamment une chronologie des rapports du compositeur avec le Québec (p. 77-81).

21. Les titres de trois des œuvres entendues au cours du festival, Treppenmusik de John Rea, Manche haben Trompinetten de Denys Bouliane et Alap et Gap de José Evangelista (page inspirée par la musique de I'Inde du Nord), parlent d'eux-mêmes.

22. Et souvent dans le seul but de combler un quota imposé par les subventionneurs. 
Québec depuis 1990, il déclare ne pas même avoir ressenti la nécessité de se rendre en Europe pour y parfaire sa formation ${ }^{23}$. Le monde musical est assez vivant au Québec, dit-il, une infrastructure solide (sociétés de concert, interprètes dévoués, radios, disques) permet d'y entendre une grande diversité de musiques. II croit que beaucoup de jeunes compositeurs choisissent d'ailleurs aujourd'hui, pour cette raison, de rester au Québec.
23. Au moment d'écrire ces lignes, il termine un doctorat en composition à l'université McGill.

\section{Existe-t-il un style québécois?}

Voilà la question centrale qui se dégage de l'ensemble de cet événement, et ce qui nous a motivé à coucher sur le papier ces quelques réflexions. Elle était au centre des interventions de Denys Bouliane sur les ondes de la radio française et, au cours de cette table ronde, il a réaffirmé avoir découvert ses racines profondément québécoises une fois à l'étranger, en Allemagne en l'occurrence ${ }^{24}$. Le fait que les Québécois soient en perpétuel questionnement, contrairement, toujours selon Bouliane, aux Allemands, se révèle fructueux sur le plan créatif. Sean Ferguson est également d'avis que les artistes Québécois, à la recherche de leur identité propre, n'évitent pas la question posée plus haut ${ }^{25}$. Selon Michel Gonneville, le caractère distinctif — un autre mot à la mode dans la classe politique... - du style québécois réside peut-être dans le fait que plusieurs des créateurs qu'il côtoie se dissocient consciemment de toute idée d'école. Isabelle Panneton renchérira en soulignant, elle aussi, le détachement des jeunes compositeurs envers ceux-là mêmes qui ont été au cœur de leur formation.

Quant à notre proximité avec les Américains, elle fascine bien sûr let irrite à l'occasion, avec souvent une pointe d'envie) nombre de compositeurs et de commentateurs français. Nul doute que plus d'un écho de la toute-puissante musique populaire américaine ait été perçu dans les œuvres présentées dans le cadre de ce festival. Or, loin de gêner les compositeurs québécois, cette présence est bel et bien revendiquée par plusieurs, notamment par Denys Bouliane qui rappelle qu'il fut guitariste rock dans sa jeunesse. En plus du contenu référentiel déjà noté, le caractère fortement ludique de plusieurs des œuvres québécoises entendues lors de l'émission de radio, et durant l'ensemble de la semaine, sera remarqué par plusieurs auditeurs, y compris les compositeurs en présence. Ce goût du faire, ce plaisir manifestement pris à l'acte compositionnel s'oppose immédiatement au sérieux, pour ne pas dire à la sévérité, des autres musiques qui figuraient au programme des divers concerts auxquels j'ai pu assister. Cela peut se comprendre. Comment rire (ou sourire) de ce qui repose sur les étagères de la mémoire musicale, comme le fait un John Rea ou une Estelle Lemire, lorsqu'on compose en France et qu'un Boulez pourrait être assis dans la salle?
24. Voir Circuit, vol. 9, n², 1998, p. 26 et suiv.

25. Et un participant de rappeler que Boulez, dans un dossier récent du Monde de la musique, niait l'existence d'une tradition française. "II n'y a pas de chaîne continue, déclarait-il. II n'y a que des compositeurs qui se donnent la main. II existe évidemment des points communs d'un compositeur à l'autre, mais il y en a autant entre Wagner et Debussy [...]". (n 229, février 1999, p. 27). Pour sa part, Serge Provost, malgré plusieurs séjours prolongés en France, déclare ne s'être jamais perçu comme un compositeur de "tradition française ». 


\section{L'impact à plus long terme}

... on se sentait [...] très importants, au centre de quelque chose de grand, d'un événement qui fera date. Sauf que, chaque fois qu'on s'est senti importants, c'était entre nous. Les Français eux, sont resté chez eux. [...] L'effervescence, les poignées de main et les compliments, c'était entre nous qu'on les échangeaient. On s'est même senti au Québec, un instant. Quel bonheur!

(Moutier, Voir, 8 au 14 avril 1999, p. 10.)

À mon avis, cette déclaration d'un jeune écrivain québécois qui faisait partie de la délégation d'auteurs invités au Salon du livre de Paris dans le cadre du Printemps du Québec ${ }^{26}$ aurait presque pu être celle, hélas, d'un des participants à Présences 99. Au-delà des applaudissements d'usage, généralement assez nourris, les compositeurs n'ont eu que peu de contacts directs avec le public venu entendre leurs œuvres. Mario Paquet s'est heureusement chargé de recueillir certains commentaires pour le bénéfice des auditeurs de Radio-Canada. Des remarques spontanées sur la qualité (doit-on comprendre «inattendue»?) des interprètes reviennent sans cesse, de même que sur la variété des musiques entendues, sur le côté très "sympathique » de la chose. Plusieurs se diront étonnés, dépaysés, parfois au point d'avoir du mal à juger les œuvres à une première écoute. La musique de Pascal Dusapin sera jugée plus "facile» d'approche. Quelques compositeurs connus, approchés par Mario Paquet, seront plus explicites. Betsy Jolas, elle-même d'origine américaine et qui a côtoyé plusieurs Québécois durant sa carrière de professeure au Conservatoire national supérieur de musique de Paris, lovera l'œuvre pour piano de Sean Ferguson (EnvoléesMarées) brillamment interprétée par Marc Couroux. Ivo Malec, lui aussi professeur au CNSMP (aujourd'hui à la retraite) et membre, en 1996, du jury du Concours national des jeunes compositeurs de Radio-Canada, sera impressionné de la qualité d'écoute de l'ensemble de ces compositeurs (mais, du fait justement de cette expérience récente, aucunement surpris, tientil à ajouter).

À la lecture de ce qui précède, on me jugera peutêtre sévère. Ou peu sensible à l'honneur qui nous a été fait. Bien au contraire. C'est pourquoi j'aurais souhaité que les journaux parisiens, qui n'en avaient que pour Dusapin, accordent davantage d'attention à notre musique ${ }^{27}$. Que les compositeurs français dont les noms circulent en ce moment se soient montrés, à l'instar d'Yves Prin et de son équipe (et de plusieurs amis du Quatuor, comme François Boyle et les compositeurs déjà nommés), véritablement intéressés à ce qui se passe ici. Mais oublions cela pour terminer plutôt sur une note positive. Grâce aux enregistrements captés sur le vif par Radio France, les œuvres des compositeurs québécois ont pu être entendues un peu partout à travers le monde; voilà, à mon avis, l'enjeu majeur d'un tel projet, et dont les retombées ne peuvent être évaluées, encore moins
26. Présences 99, qui survenait trop tôt dans le calendrier, ne s'inscrivait pas dans cet événement hautement médiatisé, ici comme en France. II s'y additionnait, plutôt.

27. Une seule critique, celle déjà citée de Gervasoni dans Le Monde, s'attardait à la participation québécoise. 
chiffrées. J'insisterai bien sûr sur la qualité de la musique entendue. Je rappellerai à cet égard le succès indéniable du concert présenté à Montréal conjointement par le NEM et l'ensemble de la SMCQ, quelques jours avant leur départ pour Paris, devant un millier de personnes (le concert était gratuit) ${ }^{28}$. Non, le public, qui, à l'ère du lecteur vidéo, du lecteur compact, de l'ordinateur personnel et de l'inforoute, n'a jamais eu autant de bonnes raisons de demeurer chez soi, n'est pas en déroute ${ }^{29}$.

Je me prends aussi, en terminant, à rêver, comme le faisait Lorraine Vaillancourt sur les ondes de Radio-Canada, à un événement semblable, produit ici, à une plus petite échelle peut-être, et qui mettrait l'accent non seulement sur les compositeurs du Québec mais aussi sur des créateurs et des ensembles étrangers de haut niveau. Des concerts gratuits, radiodiffusés à travers le monde. Le public serait de la partie, parions-le ${ }^{30}$. Remercions donc de leurs efforts soutenus tous ceux qui, Denys Bouliane, Lorraine Vaillancourt, Walter Boudreau et Yves Prin en tête, ont mis les mains à la pâte et n'ont pas compté les heures pour offrir aux compositeurs et interprètes québécois un peu d'air frais et de visibilité. Pour reprendre la formule de Denys Bouliane, "c'est un petit pas, mais un pas important». Lorraine Vaillancourt dira sensiblement la même chose lorsqu'elle confiera à Mario Paquet avoir éprouvé à Paris de la fierté. "C'est la musique de nos compositeurs [qui est jouée]. Tous ces gens [les interprètes] sont allés à Paris porter notre identité, notre culture. (La grande soirée du mercredi, 10 février 1999). Au risque de paraître un peu chauvin, le jeu en valait la chandelle.
28. Le critique Claude Gingras en témoignait le lendemain, le dimanche 24 janvier, dans La Presse (p. B 11 ).

29. Je tiens également à souligner l'intérêt du petit document Présence de la musique québécoise, consacré aux compositeurs sélectionnés et qui fut lancé lors de ce même concert et mis en vente à Paris. Des compositeurs, des musicologues et des interprètes y parlent de créateurs dont ils se sentent proches, souvent en adoptant un point de vue original et révélateur. Publiée en un temps record et dans des conditions difficiles, cet ouvrage est à lire et à consulter (pour les listes sélectives d'œuvres et la discographie).

30. Le festival Québec Musique au présent, présenté pour la deuxième année consécutive par l'Orchestre symphonique de Québec en juin dernier, en est bien la preuve.

ARCHAMBAULT, G. (20 avril 1999), "La dame de la rue Panet», Le Devoir, p. A7.

BOIVIN, J. (1996), "La classe de composition de Serge Garant, ou le sentier de la lucidité », Circuit, vol. 7, n² 2, p. 37-55.

BOULIANE, D. (1999), "À l'est de l'ouest, à l'ouest de l'est, cherchez au nord! Toile de fond d'une création musicale en pleine effervescence ", Présence de la musique québécoise. Vingt-deux portraits instantanés, Montréal, Société de musique contemporaine du Québec/Nouvel Ensemble Moderne, p. 5-10. II s'agit d'une version développée du texte paru dans le programme souvenir préparé par Radio France, p. XXII-XXIV.

BOULIANE, D. (directeur), (1999), Présence de la musique québécoise. Vingt-deux portraits instantanés, Montréal, Société de musique contemporaine du Québec/Nouvel Ensemble Moderne, 103 p.

BOULIANE, D. (1999), "L'image fécondante du silène. D'une incursion du doute, du rire et de l'ironie dans le musical: souvenirs, réflexions, "autoréflexions" ", Circuit, vol. 9, $n^{\circ} 2$, p. 25-60. 
GALAISE, S., LEDUC, D., MARCHAND, G., PERRON, P., RIVEST, J. et THIRIAR, D. (1994), "Xenakis au Québec: chronologie et repères», Circuit, vol. 5, n², p. 77-81.

GERVASONI, P. (3 février 1999), "Postmodernisme québécois à Radio France», Le Monde, p. 29.

GINGRAS, C. (24 février 1999), "SMCQ-NEM: Paris en avant-première ", La Presse, p. B 11 .

MERCIER, M.-O. (8 au 14 avril 1999), "La croisière s'amuse. Le printemps du Québec à Paris», Voir, p. 10.

Présences 99, Festival de création musicale, 29 janvier- 14 février (1999), Programme du festival, Paris, Radio France.

REA, J. (1999), "L'artiste et ses masques... Une entrevue avec le compositeur John Rea par Ferdinand Larven Niemantz (Vienne, Los Angeles) », Présence de la musique québécoise. Vingt-deux portraits instantanés, Montréal, Société de musique contemporaine du Québec/Nouvel Ensemble Moderne, p. 76-78.

\section{Source sonore}

La grande soirée du mercredi, chaîne culturelle de Radio-Canada, émissions du 10 et du 17 février 1999. Animateur: Mario Paquet. Une réalisation de Laurent Major. 